\title{
Feasibility of Ultrasound Guided Atlanto-occipital Joint Injection
}

\author{
Sun Jae Won, M.D., U-Young Lee, M.D., Ph.D. ', Sei Un Cho, M.D., Won Ihl Rhee, M.D., Ph.D. \\ Department of Rehabilitation Medicine, Yeouido St. Mary's Hospital, \\ ${ }^{1}$ Department of Anatomy, The Catholic University of Korea, College of Medicine, Seoul 150-713, Korea
}

\begin{abstract}
Objective To evaluate the feasibility of ultrasound guided atlanto-occipital joint injection.
Method Six atlanto-occipital joints of three cadavers were examined. Cadavers were placed in prone position with their head slightly rotated towards the contra-lateral side. The atlanto-occipital joint was initially identified with a longitudinal ultrasound scan at the midline between occipital protuberance and mastoid process. Contrast media $0.5 \mathrm{cc}$ was injected into the atlanto-occipital joint using an in-plane needle approach under ultrasound guide. The location of the needle tip and spreading pattern of the contrast was confirmed by fluoroscopic evaluation.

Results After ultrasound guided atlanto-occipital joint injection, spreading of the contrast media into the joint was seen in all the injected joints in the anterior-posterior fluoroscopic view.

Conclusion The ultrasound guided atlanto-occipital injection is feasible. The ultrasound guided injection by Doppler examination can provide a safer approach to the atlanto-occipital joint.
\end{abstract}

Key Words Atlanto-occipital joint, Cadaver study, Ultrasound guided injection

\section{INTRODUCTION}

The upper cervical spine often causes chronic head and neck pain. Pain originating from the atlanto-occipital joint is not so common and can be confused with various types of headache or other musculoskeletal problems, thus making diagnosis and treatment difficult.

Patients with atlanto-occipital pathology often com-

Received March 14, 2012; Accepted July 21, 2012

Corresponding author: Sei Un Cho

Department of Rehabilitation Medicine, Yeouido St. Mary's Hospital, The Catholic University of Korea, College of Medicine, 62, Yeouido-dong, Youngdeungpo-gu, Seoul 150-713, Korea

Tel: +82-2-3779-1257, Fax: +82-2-3779-2075, E-mail: shewry@hanmail. net

(c) This is an open-access article distributed under the terms of the Creative Commons Attribution Non-Commercial License (http:// creativecommons.org/licenses/by-nc/3.0) which permits unrestricted noncommercial use, distribution, and reproduction in any medium, provided the original work is properly cited.

Copyright $\odot 2012$ by Korean Academy of Rehabilitation Medicine plain of general neck pain with occipital and suboccipital pain radiating ipsilaterally, which gets aggravated by neck flexion, extension and lateral rotation. ${ }^{1}$ Another outcome can be cervicogenic headache, and some patients often complain of nausea, blurred vision, tinnitus, and insomnia. $^{1}$

The mechanism explaining pain development from the atlanto-occipital joint is variable. Degenerative osteoarthritis and acceleration-deceleration injury are likely the most common mechanisms. ${ }^{2}$ Ankylosing spondylitis, hypomobility, and destructive change of cervical spine in early rheumatoid arthritis can also contribute towards development of pain. ${ }^{3}$ However, because there are no specific pathognomic radiographic findings, ${ }^{1}$ the differential diagnosis may be difficult.

In 1989, Busch and Wilson showed clinical feasibility of steroid and local anesthetics injection into the atlanto-occipital and atlanto-axial joints in 25 patients. ${ }^{4}$ 
Dreyfuss (1994) reported clinical improvement following fluoroscopic guided atlanto-occipital joint corticosteroid injection. ${ }^{5}$ Considering these findings, atlanto-occipital joint injection can be an effective method to diagnose and treat the atlanto-occipital joint mediated pain. However, to the best of our knowledge, until date, there has been no study about atlanto-occipital joint injection after Dreyfuss' study in 1994.

Ultrasound guided injections have been vastly spreading to various medical fields, providing several advantages like, radiation-free procedure, real time inspection, and clear visualization of blood vessels.

Therefore, in the present study, we evaluated the feasi- bility of ultrasound guided atlanto-occipital joint injection on cadavers.

\section{MATERIALS AND METHODS}

This study was conducted on six atlanto-occipital joints from three fresh cadavers, with protocol approved by Institutional Review Board of our institution.

Initially, the cadaver was placed in prone position with neck flexion by setting pillow under the neck. Then the head was rotated towards the side contralateral to injection direction about 30 degrees. The mastoid process and occipital protuberance were found by palpation, and
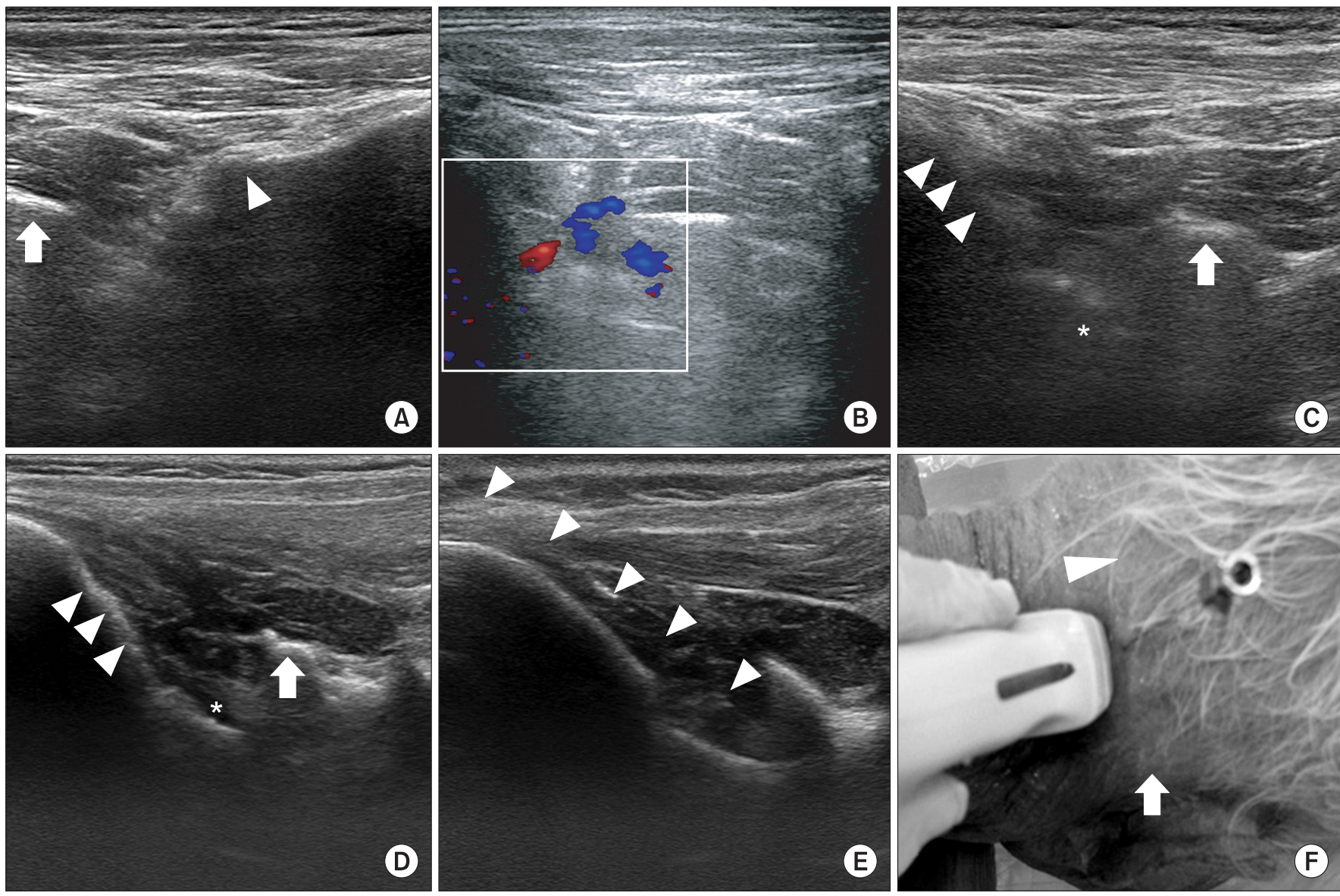

Fig. 1. Ultrasound guided atlanto-occipital joint injection. (A) In ultrasound view of living body at the longitudinal scan below the mastoid process, $\mathrm{C} 1$ transverse process (arrow) and C2-3 facet joint (arrowhead) are shown. (B) Doppler examination in living body. Vertebral artery is visualized between $\mathrm{Cl}$ transverse process and occiput. (C) In ultrasound view of living body with a longitudinal scan at the mid-point between mastoid process and occipital protuberance, atlanto-occipital joint (asterix) is seen between $\mathrm{Cl}$ transverse process (arrow) and occiput (arrowhead). (D) In ultrasound view of cadaver, same as $\mathrm{C}$, atlanto-occipital joint (asterix), $\mathrm{C} 1$ transverse process (arrow), and occiput (arrowhead) are shown. The atlanto-occipital joint is more clearly visualized than in living body. (E) In real time ultrasound guided injection, needle (arrowhead) is placed at the atlanto-occipital joint. (F) Needle insertion point is the mid-point between occipital protuberance (arrowhead) and mastoid process (arrow). 

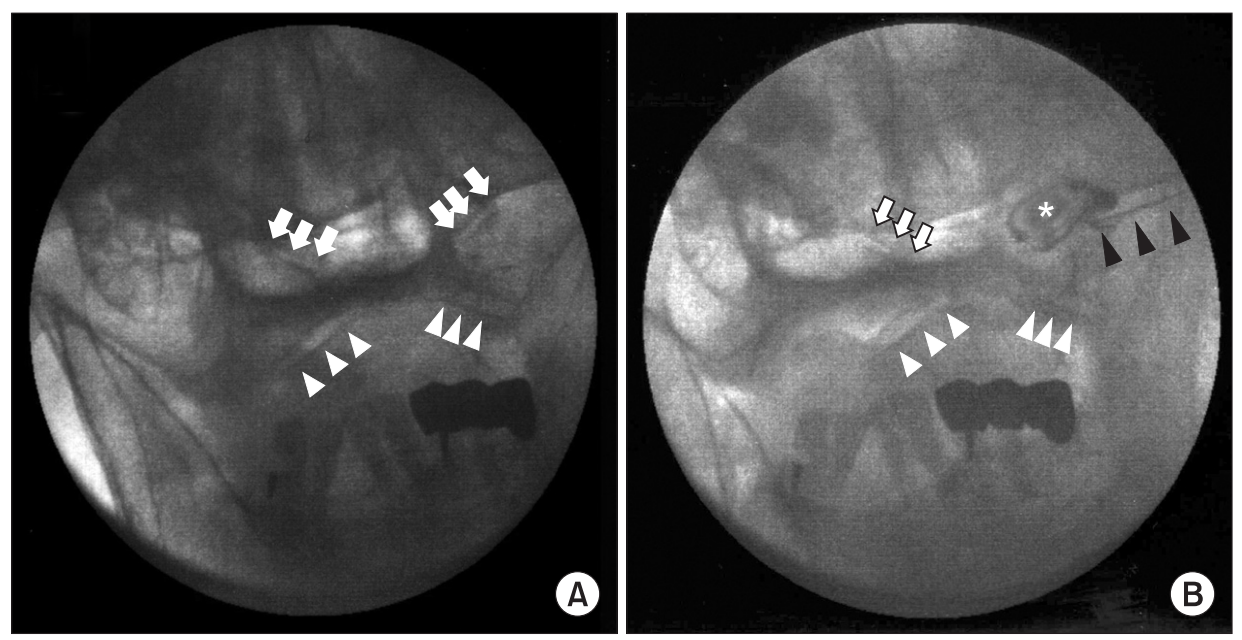

Fig. 2. Anterior-posterior fluoroscopic view of atlanto-occipital joint. (A) Before contrast media injection, bilateral atlanto-occipital joints (arrow) and atlanto-axial joints (arrowhead) are seen. (B) After contrast media injection, needle placement (black arrow) and spreading to the atlantooccipital joint (asterix) are clearly visualized.

marked. The ultrasound probe was placed in longitudinal scan to a vertical line crossing mastoid process. At this point, the probe was moved medially and laterally to check transverse process of the first cervical vertebra and C2-3 facet joint (Fig. 1-A).

In the living body, where the first cervical transverse process and C2-3 facet joint are visualized, the vertebral artery can be visualized and confirmed by Doppler examination (Fig. 1-B). The vertebral artery should be continuously checked while moving the probe medially. Before the midpoint between mastoid process and occipital protuberance, the vertebral artery runs anterior to spinal cord; thus, it disappears from the ultrasound view. After disappearance of vertebral artery, keeping the probe moving medially, the atlanto-occipital joint was found at the midpoint between mastoid process and occipital protuberance (Fig. 1-F).

In a cadaver, the atlanto-occipital joint was clearly visualized (Fig. 1-D). However, in a living body, the atlantooccipital joint line may be less clear (Fig. 1-C). Therefore, the probe angle should be adjusted, and compression with probe is necessary to get clearer imaging.

After confirming the atlanto-occipital joint by ultrasound view, the needle tip was inserted at the midpoint between mastoid process and occipital protuberance, and advanced to the target point using in-plane approach under real-time ultrasound guidance (Fig. 1-E, F). After the needle tip was placed at the atlanto-occipital joint in ultrasound view, we confirmed its position by anteroposterior view of fluoroscopy, and subsequently injected 0.5 cc of contrast media, and checked its spread near the joint space (Fig. 2).

\section{RESULTS}

A total of 6 atlanto-occipital joints from 3 cadavers were evaluated. The needle tip was placed at the lateral border of atlanto-occipital joint, and the contrast spreading to the joint space was confirmed in all the cases.

\section{DISCUSSION}

In this study, we demonstrated the feasibility of ultrasound-guided injection and the potentiality of ultrasound-guided injection for accurate needle tip placement to the atlanto-occipital joint. If physiatrists observe the principle of avoiding blood vessels with Doppler examination and close monitoring of needle tip placement, the procedure will be safe and accurate.

To perform an appropriate injection at the atlantooccipital joint, careful review and detailed anatomic consideration is necessary because the atlanto-occipital joint has a unique anatomic feature. The atlas, first cervical vertebra, has a wide ring shape structure and no vertebral body. Its spinous process is hardly noticeable and the anterior and posterior tubercles of the transverse process are not clearly separated. ${ }^{6}$ In the antero-lateral part of ring, the atlas articulates with the occipital condyle and makes the atlanto-occipital joint. Unlike other cervical facet joints, its joint line lies transversely, making an acute joint angle between atlas and occiput. This acute joint angle presented some technical difficulty in advancing the needle to the target point. Thus, operators should closely monitor the needle tip advancement during procedures. If the needle tip is appropriately located 
in the articular space and the absence of vessels around the needle tip is confirmed with Doppler examination, the injection does not cause adjacent tissue swelling; this may represent the successive intra-articular injection.

Important structures near the atlanto-occipital joint include vertebral artery, carotid artery, jugular vein, spinal cord and $\mathrm{Cl}$ spinal nerve. Among them, the vertebral artery runs anterior to the atlanto-occipital joint and enters the foramen magnum. The carotid artery and jugular vein run more antero-laterally, ${ }^{1}$ and enter the carotid and jugular foramen. The vertebral artery is most closely located to the joint, so the clinician should take care to avoid damage to the vertebral artery during the atlanto-occipital injection. Dreyfuss et al. ${ }^{5}$ using fluoroscopy guided atlanto-occipital joint injection, chose the mid-point between mastoid process and occipital protuberance for the injection site because of the location of the vertebral artery. In our study, based on human ultrasound examination of vertebral artery, the mid-point between mastoid process and occipital protuberance was chosen as the injection site, because there was no vertebral artery in ultrasound view.

Some investigators reported vertebral artery anomalies in the atlantoaxial area. ${ }^{7,8}$ They reported 3 types of such variation: (1) vertebral artery running in the spinal canal below $\mathrm{Cl}$; (2) fenestration of the vertebral artery above and below $\mathrm{Cl}$; and (3) posterior inferior cerebellar artery originating from the $\mathrm{Cl} / 2$ level. Fortunately, in our case, the vertebral artery was found to run anterior to the spinal cord before our injection site. So, ultrasound guided atlanto-occipital injection can be performed regardless of vertebral artery variation. Furthermore, Doppler examination visualizes the vertebral artery in real time, so even with unexpected anatomic variation; safe injection can be performed, with ready application to the clinical setting.

The atlanto-occipital joint can cause neck pain, ipsilateral occipital area pain and cervicogenic headache. Due to its diagnostic difficulty, there has been no published literature about the prevalence of atlanto-occipital joint pathology. Suggested causes include trauma or degenerative joint diseases. Depending upon pathogenesis, atlanto-occipital joint mediated pain can have a variety of pain and associated symptoms. For example, in whiplash injury, patients may show symptoms of post-concussion syndrome, dizziness and poor concentration, but in degenerative joint disease, patients complain only of neck and occipital area pain. ${ }^{1}$ Thus, careful review of associated symptoms is useful for differential diagnosis. Among imaging studies, coronal and sagittal reconstruction CT scan is the most effective approach to evaluate atlantooccipital joint as it can show joint space narrowing, subchondral sclerosis, ostephyte or cyst formation. ${ }^{9}$ In bone scan, increased uptake can be seen in atlanto-occipital joint, ${ }^{10}$ but this is not a definite diagnostic finding. Even though imaging studies such as simple $\mathrm{X}$ ray, which covers anterior-posterior, lateral and open mouth view, CT scan and MRI, are useful to rule out other similar diseases, the final diagnosis is usually based on history and physical examination. The atlanto-occipital joint injection is not only valuable for symptom reduction, but also useful for the definite diagnosis.

In the acute stage within 2 weeks, the atlanto-occipital joint mediated pain can improve with medication such as NSAIDs, muscle relaxant, tricyclic antidepressant, oral steroids and physical therapy. When symptoms cannot be controlled in spite of these treatments, a more invasive approach may be necessary to prevent development of the chronic stage. ${ }^{1}$ In some limited cases, surgical treatment like occipito-cervical fusion may be useful, ${ }^{9}$ but it is risky and not cost effective. Corticosteroid injection into the cervical facet joints has been proven to be an effective and safer approach in many clinical trials. ${ }^{11,12}$ The atlanto-occipital joint injection was also proved to be effective and safe. ${ }^{3,4}$ In all these trials for atlanto-occipital joint injection, the approach to the target area was performed under fluoroscopic guidance. Fluoroscopic guided injection, while useful to confirm correct needle placement, has several disadvantages such as radiologic exposure, requirement of complex equipment, and high cost. Nowadays, ultrasound guided injection has been proven to be effective and safe. ${ }^{13-15}$ Ultrasound has many advantages, including real time visualization of target and near structure, no radiologic exposure, requirement of simple equipment and cost effectiveness. In particular, ultrasound can clearly show vascular structures by Doppler examination, enabling the operator to advance the needle by avoiding the vessels. Therefore, it may be a safer injection than fluoroscopic guided approach for avoiding damage to vascular structures. In our study using the ultrasound guide, in all the 6 attempts, contrast spreading was confirmed by fluoroscopy. Ultrasound guided atlanto-occipital injection can be expected to be as effective as fluoroscopic guided injection. Given that atlanto- 
occipital joint mediated pain is not common and difficult to diagnose, it is proposed that this injection technique could prove to be very useful.

There are several limitations in our study. We tested the injection only in cadavers, so we could not evaluate real clinical outcomes and potential adverse effects. Possible complications include allergic response, vascular spreading of injected material, infection of the injection site and subdural spreads in cervical facet joint injection. ${ }^{11}$ Cervical spinal intervention may cause several complications such as infection, seizure, total spinal block, nausea, vomiting, headache, respiratory distress, hypotension and spinal cord injury. Among these, serious complications are usually caused by intravascular or intradural injection. However, in ultrasound-guided injections with in-plane approach, the operator can sensitively detect the vascular system and monitor the placement of the needle tip, minimizing the risk of serious complications. Another limitation is the small number of cadavers. However, we confirmed correct spreading of contrast media via fluoroscopic view in all cases, further study dealing with more cases is needed to reinforce our findings. Finally, we tested ultrasound-guided injection accuracy only by fluoroscopic view, and did not dissect cadaver. We chose fluoroscopy confirmed method because it is most commonly used for routine spinal interventions and is perceived as the standard protocol in clinical setting. While fluoroscopy is the routine method of spinal intervention, dissection of cadaver is more accurate and can assess damage of nearby structures.

To the best of our knowledge, this is the first study about ultrasound-guided atlanto-occipital joint injection. We demonstrated its feasibility by fluoroscopic examination. Based on this cadaveric study, we anticipate that further research dealing with management of atlanto-occipital joint pathology will be undertaken.

\section{CONCLUSION}

We demonstrated the feasibility of ultrasound-guided atlanto-occipital joint injection in the cadaveric study.

\section{ACKNOWLEDGEMENTS}

The donated cadavers for this research was supported by Catholic Institute for Applied Anatomy, College of
Medicine, The Catholic University of Korea. We thank the spirits of all people who donated their bodies.

\section{REFERENCES}

1. Ogoke BA. The management of the atlanto-occipital and atlanto-axial joint pain. Pain Physician 2000; 3: 289-293

2. Meleger AL, Krivickas LS. Neck and back pain: musculoskeletal disorders. Neurol Clin 2007; 25: 419-438

3. Halla JT, Hardin JG, Vitek J, Alarcon GS. Involvement of the cervical spine in rheumatoid arthritis. Arthritis Rheum 1989; 32: 652-659

4. Busch E, Wilson PR. Atlanto-occipital and atlantoaxial injections in the treatment of headache and neck pain. Reg Anesth 1989; 14: 45

5. Dreyfuss P, Rogers J, Dreyer S, Fletcher D. Atlanto-occipital joint pain. A report of three cases and description of an intraarticular joint block technique. Reg Anesth 1994; 19: 344-351

6. Menezes AH, Traynelis VC. Anatomy and biomechanics of normal craniovertebral junction (a) and biomechanics of stabilization (b). Childs Nerv Syst 2008; 24: 1091-1100

7. Tokuda K, Miyasaka K, Abe H, Abe S, Takei H, Sugimoto S, Tsuru M. Anomalous atlantoaxial portions of vertebral and posterior inferior cerebellar arteries. Neuroradiology 1985; 27: 410-413

8. Uchino A, Saito N, Watadani T, Okada Y, Kozawa E, Nishi N, Mizukoshi W, Inoue K, Nakajima R, Takahashi M. Vertebral artery variations at the C1-2 level diagnosed by magnetic resonance angiography. Neuroradiology 2012; 54: 19-23

9. Yoshihara H, Kepler C, Hasegawa K, Rawlins BA. Surgical treatment for atlantooccipital osteoarthritis: a case report of two patients. Eur Spine J 2011; 20 Suppl 2: S243-247

10. Valdez DC, Johnson RG. Role of technetium-99m planar bone scanning in the evaluation of low back pain. Skeletal Radiol 1994; 23: 91-97

11. Falco FJ, Erhart S, Wargo BW, Bryce DA, Atluri S, Datta S, Hayek SM. Systematic review of diagnostic utility and therapeutic effectiveness of cervical facet joint interventions. Pain Physician 2009; 12: 323-344

12. Manchikanti L, Singh V, Falco FJ, Cash KM, Fellows B. Cervical medial branch blocks for chronic cervical 
facet joint pain: a randomized, double-blind, controlled trial with one-year follow-up. Spine 2008; 33: 1813-1820

13. Pekkafahli MZ, Kiralp MZ, Basekim CC, Silit E, Mutlu H, Ozturk E, Kizilkaya E, Dursun H. Sacroiliac joint injections performed with sonographic guidance. J Ultrasound Med 2003; 22: 553-559

14. Greher M, Scharbert G, Kamolz LP, Beck H, Gustorff B, Kirchmair L, Kapral S. Ultrasound-guided lumbar facet nerve block: a sonoanatomic study of a new methodologic approach. Anesthesiology 2004; 100: 1242-1248

15. Galiano K, Obwegeser AA, Bodner G, Freund MC, Gruber H, Maurer H, Schatzer R, Fiegele T, Ploner F. Ultrasound-guided facet joint injections in the middle to lower cervical spine : a CT-controlled sonoanatomic study. Clin J Pain 2006; 22: 538-543 